\title{
Transatlantica
}

Revue d'études américaines. American Studies Journal

Hélène Le Dantec-Lowry, De l'esclave au Président. Discours sur les familles noires aux États-Unis, Paris, CNRS Editions, 2010

\section{Guillaume Marche}

\section{(2) OpenEdition}

Journals

Édition électronique

URL : http://journals.openedition.org/transatlantica/5377

DOI : 10.4000/transatlantica. 5377

ISSN : $1765-2766$

Éditeur

AFEA

Référence électronique

Guillaume Marche, «Hélène Le Dantec-Lowry, De l'esclave au Président. Discours sur les familles noires aux États-Unis, Paris, CNRS Editions, 2010 », Transatlantica [En ligne], 1 | 2011, mis en ligne le 03 janvier 2012, consulté le 29 avril 2021. URL : http://journals.openedition.org/transatlantica/5377 ; DOI : https://doi.org/10.4000/transatlantica.5377

Ce document a été généré automatiquement le 29 avril 2021

\section{(c) (1) $\odot$}

Transatlantica - Revue d'études américaines est mis à disposition selon les termes de la licence Creative Commons Attribution - Pas d'Utilisation Commerciale - Pas de Modification 4.0 International. 


\title{
Hélène Le Dantec-Lowry, De l'esclave au Président. Discours sur les familles noires aux États-Unis, Paris, CNRS Editions, 2010
}

\author{
Guillaume Marche
}

1 Préfacé par le sociologue Loïc Wacquant, l'ouvrage d'Hélène Le Dantec-Lowry propose une étude historiographique approfondie analysant les regards portés sur les familles noires aux États-Unis des débuts de l'esclavage jusqu'à aujourd'hui. Le sujet embrassé est encore plus complexe qu'il n'y parait, puisqu'il ne s'agit pas simplement de dérouler le récit linéaire de l'évolution d'un ensemble de représentations historiquement déterminées. Hélène Le Dantec-Lowry met ses lecteurs en présence d'un objet d'étude multidimensionnel qui lui donne la possibilité d'explorer l'histoire afro-américaine dans son ensemble. Il s'agit en particulier d'interroger les termes mêmes de la recherche : que signifient par exemple les termes « noir », « afro-américain », " africain américain »? Peut-on ou doit-on encore parler d'identification raciale, ou bien faut-il dépasser la catégorie de "race" pour envisager la population américaine d'origine africaine en termes socio-économiques? En quoi l'étude de pratiques privées telles que les configurations et les structures familiales permet-elle de saisir les questions politiques qui sont posées à ce groupe ethno-racial?

2 L'auteure part d'une évolution en trois phases : après les avoir dénigrées comme étant une déviation pathologique ou dangereuse du modèle blanc dominant, les discours sur les familles noires se sont employés à reconnaître en elles un modèle alternatif marqué par sa différence vis-à-vis du schéma de la famille nucléaire et facteur de résilience culturelle pour les Noirs. Dans un troisième temps, les familles noires ont été envisagées en termes de complexité et de diversité, dont une opposition binaire famille nucléaire blanche / famille noire élargie ne suffit à rendre compte. Cette évolution s'écrit en parallèle avec trois grands moments de l'histoire afro-américaine - le long temps de l'esclavage du début du XVII ${ }^{\mathrm{e}}$ siècle au milieu du XIX ${ }^{\mathrm{e}}$ siècle, la migration du 
Sud vers le Nord de la fin de la Reconstruction aux années 1960, la situation d'infériorité économique résultant de la ségrégation résidentielle de facto des années 1960 à aujourd'hui - qui constituent l'architecture de l'ouvrage. Le livre croise ces deux temporalités pour contextualiser la première par rapport à la seconde.

3 Ainsi Hélène Le Dantec-Lowry montre-t-elle non seulement que l'on n'écrit pas sur les familles noires de la même manière selon l'époque, mais également que cette écriture constitue un important enjeu politique: on ne développe pas le même discours politique sur le statut des Afro-Américains selon que l'on décrit les familles noires esclaves comme étant totalement soumises à la volonté des propriétaires, ou bien comme constituant des espaces de résistance. Mettre en avant les racines culturelles africaines supposées des configurations familiales noires au tournant $d u x^{e}$ siècle permet d'affirmer une certaine fierté noire, voire une position nationaliste, tandis que l'étude des facteurs sociaux exogènes conditionnant les différences entre les familles noires et blanches, et entre les familles noires elles-mêmes - comme le firent notamment les sociologues W.E.B. DuBois et Franklin Frazier - reflète l'expression d'une posture plutôt intégrationniste ouverte sur le progrès social des Noirs aux ÉtatsUnis.

4 L'ouvrage met en outre l'accent sur la rupture historiographique représentée par les années 1960 et les changements induits par le mouvement pour les droits civiques. Dans le contexte d'un tournant épistémologique où se pratiquait une histoire « vue d'en bas », le point de vue des Afro-Américains était privilégié et ce, d'autant plus que les politiques intégrationnistes permettaient à davantage de Noirs d'accéder à l'enseignement supérieur et à la recherche. Hélène Le Dantec-Lowry analyse notamment les positionnements antagonistes dans le sillage du rapport sur la famille afro-américaine rédigé par Daniel P. Moynihan pour l'administration Johnson et publié en 1965, selon lequel les familles noires seraient dysfonctionnelles - et facteurs de pauvreté - car matriarcales. L'ouvrage contextualise le débat opposant les tenants de l'assimilation des Noirs au modèle américain - c'est-à-dire blanc - aux tenants d'une identité afro-américaine spécifique. L'enjeu à la fois politique et épistémologique est alors de savoir s'il faut insister sur les déterminismes conditionnant la différence entre les configurations familiales noires et blanches, ou bien affirmer que les structures familiales reflètent la capacité d'action (agency) des Afro-Américains. Hélène Le DantecLowry montre ainsi que la construction des familles noires comme objet d'étude est tributaire d'orientations méthodologiques ou théoriques, et même de choix thématiques qu'il convient d'historiciser et qui se prêtent à une interprétation politique.

5 Dans chacune des trois parties de l'ouvrage, l'auteure met en avant le caractère heuristique de la prise en compte croisée des notions de genre et de classe. Envisager les rapports entre les sexes ou entre les classes au sein du groupe afro-américain permet en effet de sortir l'étude des familles noires d'une comparaison duelle entre familles noires et blanches, et, plus largement, de dégager l'analyse de la question raciale aux États-Unis de l'opposition binaire Blancs / Noirs. De la même manière, les monographies se concentrant sur une région explorent souvent des terrains moins connus et offrent ainsi une vision plus complexe des conditions sociales réelles dans lesquelles se constituent les familles noires - qu'il s'agisse de l'expérience de l'esclavage, de la migration ou de la pauvreté au sein des ghettos. L'un des points centraux de l'ouvrage consiste, d'une part, à affirmer que les discours sur les familles 
noires tombent dans le schématisme lorsqu'ils totalisent « la » famille noire et, d'autre part, à montrer que les discours tendant à pathologiser les familles noires se sont construits précisément au moment où de plus en plus de familles américaines échappaient au modèle nucléaire censé incarner la norme: la désignation de la déviance supposée des familles noires servit ainsi à renforcer la norme incarnée par une famille blanche idéalisée, au moment même où elle était fortement remise en cause dans les faits.

6 Le livre suggère donc l'idée d'un progrès dans la précision et dans l'appréhension de la complexité des enjeux. Par exemple, depuis les années 1990, des monographies portant notamment sur des régions frontalières ont montré que certaines familles élargies avaient maintenu des échanges Nord-Sud - échanges épistolaires et visites familiales ou favorisé des migrations à rebours du Nord vers le Sud, si bien que l'expérience migratoire n'est en réalité ni uniforme ni univoque : sa compréhension s'affine grâce à la prise en compte de variations temporelles et spatiales. L'auteure consacre des pages particulièrement intéressantes à la question des pratiques mémorielles, volontaires ou non - telles les habitudes culinaires - au sein des familles noires : ces pratiques sont souvent le fait des femmes, qui jouent un rôle de passeuses ou de relais mémoriels. Mais elle présente aussi les familles noires comme un chantier de recherche qui n'est jamais complètement abouti. En particulier, les discours scientifiques s'enrichissent de leur interdisciplinarité: le dialogue entre histoire et sociologie est constamment en filigrane. De même, l'auteure montre comment les discours émanant de journalistes ou de décideurs politiques s'ancrent dans des contextes particuliers - telle la « culture de la dépendance » supposée des familles noires monoparentales vis-à-vis de l'aide sociale, au moment même, les années 1980, où l'État s'en désengageait fortement - tandis qu'ils créent des conditions politiques qui influent à leur tour sur les discours scientifiques. Hélène Le Dantec-Lowry invite ainsi à ne " pas essentialiser le concept de 'famille' » (197) lorsqu'il s'agit d'envisager le rôle des femmes et des mères, afin de ne pas naturaliser le modèle de la famille nucléaire. Outre les facteurs de genre et de classe, il convient pour ce faire de prendre en compte la diversité des lieux, la succession des générations et l'interaction entre elles, ainsi que le travail de mémoire.

7 En abordant l'historiographie des Afro-Américains sous l'angle des familles noires, Hélène Le Dantec-Lowry prend donc la famille comme un bon indicateur de la persistance de la pertinence des identités ethno-raciales : à condition qu'elle ne soit pas prise dans un sens étroit, totalisant et univoque, la notion de "race " permet, en injectant un point de vue culturel, de rendre compte de l'expérience afro-américaine de manière plus riche que ne sauraient le faire les approches strictement socioéconomiques. Cet ouvrage propose donc non seulement une synthèse très richement documentée de l'état de la littérature historique et sociologique sur le sujet, mais il soulève d'importantes questions politiques, théoriques et méthodologiques. On peut regretter la présence d'anglicismes dans la rédaction et certaines interprétations sont sans doute discutables - notamment l'idée selon laquelle l'usage des termes "désorganisation» ou "démoralisation » serait nécessairement péjoratif dans la littérature sociologique de l'École de Chicago (93-94 en particulier) - mais l'ouvrage propose à la fois des questionnements épistémologiques approfondis, de la part d'une spécialiste reconnue des études afro-américaines, et des mises en contexte de l'histoire des États-Unis et de l'histoire des Noirs qui le rendent abordable et stimulant pour les lecteurs non spécialistes de ces deux champs. 
INDEX

Thèmes : Recensions

\section{AUTEUR}

GUILLAUME MARCHE

Université Paris-Est Créteil 